\title{
El cambio curricular de las carreras de la Licenciatura en Biología en la Facultad de Ciencias Naturales y Museo (UNLP).
}

The curriculum change of the careers of Bachelor degree in Biology of the Facultad de Ciencias Naturales y Museo (UNLP).

Carrión Cristian A.

https://orcid.org/0000-0001-5365-4858 carrioncristian@hotmail.com

Instituto de Ciencias Polares | Ambientales y Recursos Naturales IUNTdF.

\section{Posadas Paula}

https://orcid.org/0000-0002-2396-2283

posadas.paula@gmail.com

Facultad de Ciencias Naturales y Museo I UNLP.

\author{
Mónica Ros \\ https://orcid.org/0000-0002-1430-6196 \\ monica.ros@presi.unlp.edu.ar \\ Facultad de Periodismo y Comunicación \\ Social I UNLP.
}

\section{RESUMEN}

Las carreras de Biología de la FCNyM de la UNLP poseen los mismos planes de estudios desde hace al menos 34 años. La ausencia de espacios institucionalizados y regulares de discusión respecto del perfil de formación, ha contribuido a la modificación de contenidos en los espacios fragmentarios de las cátedras, sin una mirada de conjunto. Es a partir del año 2008 que ha sido posible un proceso continuo de cambio curricular con la participación de todos los actores de esta unidad académica. En este trabajo se desarrolla una sistematización analítica y crítica sobre los antecedentes curriculares y los sujetos y discursos que predominan en el proceso actual de cambio curricular.

\section{ABSTRACT}

The Biology careers of the FCNYM (UNLP) have had the same curricula for at least 34 years. The absence of institutionalized and regular spaces of discussion regarding the training profile, has resulted in the modification of content in the fragmented spaces of the academic Chairs, thus lacking of an overaII look. It is from 2008, that a continuous process of curriculum change has been possible with the participation of all the actors of this academic unit. This work develops an analytical and critical systematization on the curricular background and the subjects and discourses that predominate in the current process of curriculum change.

PALABRAS CLAVE Cambio Curricular, FCNyM, Biología, Sistematización Crítica
KEY WORDS

Curriculum Change, FCNyM, Biology, Critical Systematization 


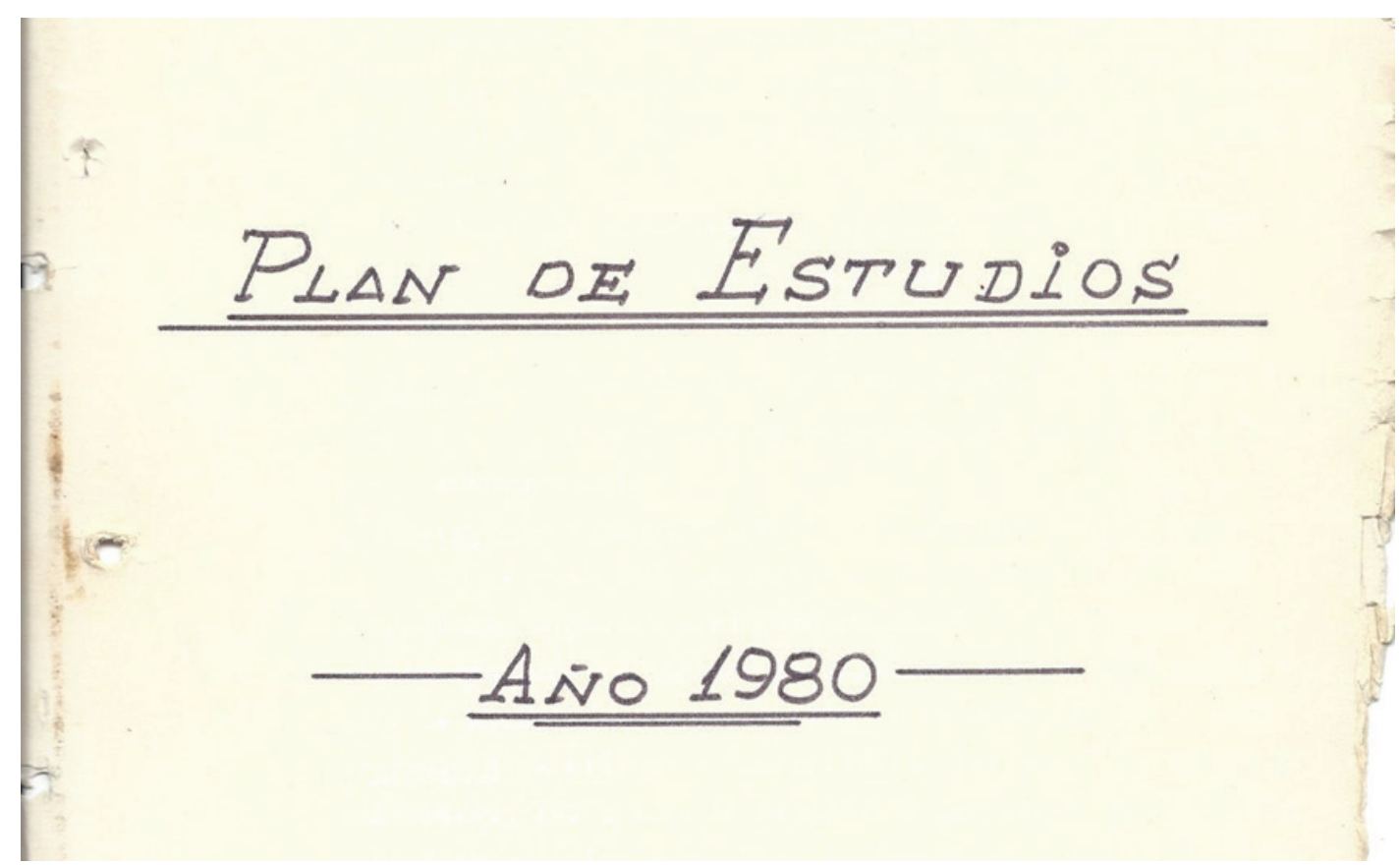

La imagen inicial corresponde a la carátula manuscrita del expediente 1000-16822-1979-000 de la FCNyM que dio origen a los planes de estudios vigentes de las cuatro carreras de Licenciatura en Biología.

\section{INTRODUCCIÓN}

Este ensayo surge de la propia trayectoria docente, y específicamente Este ensayo surge de la propia trayectoria docente, y específicamente en el marco de la participación en el proceso del cambio curricular desarrollado en las carreras de Licenciatura en Biología de la Facultad de Ciencias Naturales y Museo (FCNyM). Aunque en el análisis documental y reflexivo de estas experiencias se buscó un grado útil de extrañamiento, es importante destacar que este trabajo de sistematización estará articulado por la experiencia personal desarrollada en distintos ámbitos de discusión-elaboración desde hace 10 años en la FCNyM (Autor, 2018: tesis).

En la construcción de los currículos de las carreras de Biología de la FCNyM, ha prevalecido el criterio de reproducción de las tradiciones 
científicas del Museo de La Plata que poseen una fuerte impronta "inventarista" de la naturaleza. Según el ideario de la comunidad de la FCNyM, una parte importante de la formación que tienen los estudiantes trata sobre la revisión descriptiva de una fracción de la biodiversidad, con frecuente reducción a los aspectos sistemáticos. Aunque durante el proceso de discusión curricular esta dimensión ha sido un problema emergente muy relevante, y los documentos se han elaborado con un perfil más integral del campo de la biología, un cambio de planes de estudios no es suficiente per se para producir una transformación en la perspectiva del campo disciplinar y de la enseñanza en la comunidad académica.

La Facultad posee actualmente siete carreras de grado, cuatro de las cuales son Licenciaturas en Biología con las orientaciones Paleontología, Botánica, Zoología y Ecología. Los planes de estudios vigentes datan de entre 1980 y 1987, por lo que hace al menos 34 años que estos planes no han sido sometidos a un análisis sistemático de contenidos, estructuras y sentidos. Esto ha ocurrido a pesar de que las ciencias biológicas se cuentan entre las disciplinas que mayor cantidad y calidad de conocimiento han desarrollado en ese mismo período. Sólo como ejemplos se pueden citar algunos de los avances más destacados en el campo de la biología: el desarrollo y difusión de las técnicas de biología molecular, el concepto de biodiversidad y las bases modernas de la etología, entre decenas de otras innovaciones. Aunque estos temas se han conformado como sub-campos en la biología, y fragmentariamente han sido incluidos en algunas asignaturas, no han podido ser integrados como parte de un currículo reflexionado que los posicionara expresamente en espacios o áreas curriculares claramente definidos. Por la imposibilidad durante décadas de una intervención institucional curricular integral, los esfuerzos de organizar y actualizar contenidos han sido restringidos a la voluntad individual de algunos docentes de la institución. En la lógica de las instituciones universitarias, la coexistencia de diversas posiciones y grupos poco articulados o de bajo grado de interrelación, dificultan los procesos complejos y prolongados de discusión que los currículos requieren. Estos procesos deben asegurar una relectura de los perfiles de formación que habiliten lógicas de transformación curricular que van más allá de la incorporación de conocimientos más actualizados.

Una de las consecuencias de tener planes de estudios de cuatro décadas es la acumulación desarticulada de contenidos que habría colaborado en prolongar el promedio de egreso de los alumnos entre otras variables y condiciones. Como ejemplo, el promedio de años de egreso (duración real de las carreras) de la orientación en Zoología es de 11 años, en contraposición con otras carreras similares en Argentina que tienen un promedio de egreso de 7 años. Sobre la base de las estadísticas brindadas por la Secretaría Académica de la Facultad, el promedio de egreso de los estudiantes desde 1990 se incrementa en un año cada cinco años que transcurren (figura 1). Entonces, si se continúa con esta tendencia, los ingresantes 2020 egresarían en pro- 
medio en 2032. Por supuesto que este promedio tiene una dispersión importante, pero muestra una tendencia crítica de la cual la institución debe dar cuenta.

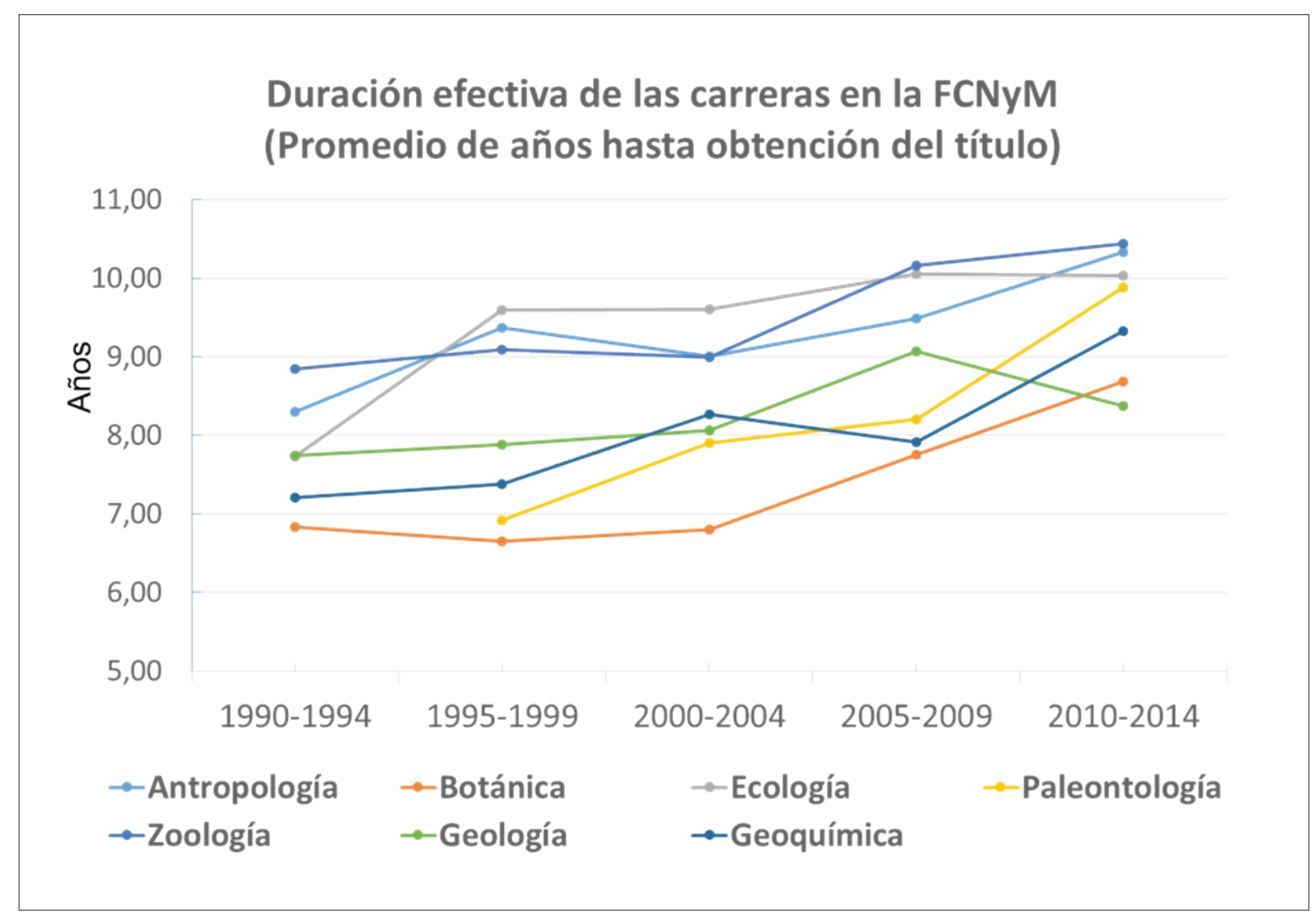

Figura 1: Duración efectiva promedio de las carreras de la FCNyM. Fuente: Secretaría Académica FCNyM.

En relación a otras carreras de Biología en Argentina, en la FCNyM los planes de estudios tienen una fuerte influencia de una tradición naturalista desarrollada en los comienzos del Museo de La Plata. El propósito de formación de las primeras generaciones de Doctores en Ciencias Naturales fue la de cubrir las necesidades de mantenimiento y expansión de las colecciones del Museo de La Plata y la necesidad social de conocer los recursos naturales de que disponía el país. Aunque en 1949 la formación de grado pasa de la órbita del Museo Facultad de Ciencias Naturales a la de la Facultad de Ciencias Naturales y Museo, esa influencia llega hasta nuestros días poniendo en tensión distintos paradigmas en las ciencias naturales y las expectativas de las nuevas generaciones de estudiantes.

En relación a otras carreras de Biología en Argentina, en la FCNyM los planes de estudios tienen una fuerte influencia de una tradición naturalista desarrollada en los comienzos del Museo de La Plata. 
Al analizar la trayectoria académica-institucional desde el Museo de 1887 a la Facultad en la actualidad se pueden notar al menos tres ejes de transformación: el estilo de enseñanza, la matrícula y los propósitos de formación. Brevemente se ensayarán algunas ideas sobre estos ejes que han conducido a los planes de estudios vigentes y posiblemente a los futuros.

Desde el siglo XVIII las universidades occidentales desarrollan la enseñanza-investigación científica a partir de dos modelos que posteriormente fueron llamados: napoleónico (escuela francesa) y humboldtiano (escuela alemana) (Burton, 1997). Estos enfoques se podrían sintetizar pensando al conocimiento como medio o al conocimiento como fin suficiente. También se diferencian en el modo de producción del conocimiento, siendo restringido a los "especialistas" en la universidad napoleónica, en oposición a la generación de conocimiento a partir de la relación tutor-alumnos (mayéutica socrática) en la universidad humboldtiana. Es en esta última corriente que el Museo de La Plata se desarrolla inicialmente. La influencia de la escuela alemana se manifestó fuertemente en la libertad académica, tanto de los estudiantes en elegir sus trayectos de formación como de los profesores en el dictado de los cursos (García, 2004: 108).

En el ámbito de la enseñanza unos pocos alumnos coexistiendo con sus profesores desarrollaban el proceso de enseñanza-aprendizaje desde la práctica. Desde 1920 hasta 1932, sólo 12 alumnos se entrenaron en el Museo de La Plata (Teruggi, 1994: 134). Esta forma de aprendizaje de estilo artesanal solo era posible con una relación maestro/aprendiz baja.

En 1890 se funda la Universidad de La Plata, y en 1906 se dicta la nacionalización de la misma, con un fuerte predominio de la investigación como su fundador Joaquín V. González expresó:

"... conocen mis ideas sobre la labor universitaria, ella es de investigación en primer término y de enseñanza o sea de extensión en segundo término (...) Una Universidad es un laboratorio de observación y experiencia de la vida del medio en que actúa; y si no es así, carece de razón de existir (...) Y esta es una Universidad científica, en aquel sentido, de preferencia por el aspecto experimental del estudio en todas sus disciplinas". Aquí Joaquín V. González plantea una inflexión epistemológica que el Museo de La Plata, por su inercia naturalista no pudo empezar a incorporar hasta mediados del siglo XX: el pasaje de una ciencia descriptiva distante de la naturaleza (ciencia de las colecciones) a una ciencia intervencionista y experimental de la naturaleza. 
Durante toda la historia de las carreras del Museo y luego de la Facultad, el número de materias se ha ido incrementando. Desde las 14 materias originales cursadas durante cuatro años para obtener el título de Dr. en Ciencias Naturales de principios de siglo XX, 112 años después se tiene alrededor de 26 materias a cursar en un plan de cinco años pero que presenta un promedio de duración efectiva de 10 años para obtener el título de Licenciado. Luego, se requieren otros 5 años aproximadamente para obtener el título de Doctor. Esta tendencia en el incremento de las materias de grado es un fenómeno que ocurre en todos los campos del conocimiento científico, derivado esto de la acumulación exponencial de información y del creciente grado de especialización requerido para abordarlo.

Siguiendo la tendencia iniciada en la década del '40 con el incremento de las posibilidades de acceso a la enseñanza universitaria, producto del crecimiento de la clase media argentina y de la fuerte expansión de la explotación de los recursos naturales territoriales, la matrícula de las carreras de la Facultad Museo se fue incrementando hasta la década de los '90. Este proceso de masificación tuvo como consecuencia un progresivo cambio en el estilo de enseñanza, desde el modelo maestro-aprendiz (humboldtiano) experimentado en el Museo desde 1909 hasta aproximadamente 1933 con solo una docena de alumnos, hasta su coexistencia con un modelo de enseñanza masiva (con semblanzas en la perspectiva de universidad napoleónica) con aproximadamente 3000 alumnos en la FCNyM de hoy. Esto hace que, en el diseño curricular de los planes de estudios, se suman complejidades relativas a los aspectos logísticos y pedagógicos para el manejo de matrículas que desafían a las formas de enseñanzas tradicionales.

Es interesante contrastar cómo han ido cambiando los propósitos de formación desde el Museo a la Facultad. Desde su nacimiento en 1909 hasta aproximadamente 1930, las carreras tenían como propósito poder dar continuidad y proyección a la institución científica. La idea era formar a las siguientes generaciones de naturalistas que seguirían haciendo crecer las colecciones e investigaciones del Museo. En los actuales planes de estudios, los aspectos inventaristas siguen siendo relativamente predominantes en comparación con otras carreras similares, lo cual constituye una característica distintiva de la institución. Desde la década del 50, los graduados han tenido una creciente oferta de inserción laboral en el campo de la investigación básica en el Consejo Nacional de Investigaciones Científicas y Técnicas (CONICET), que tiene en general un perfil experimental de la ciencia. Los graduados de las carreras de Biología también se insertan en ámbitos de gestión ambiental, docencia y actuación profesional. Estos nuevos campos de aplicación de los graduados demandan planes de estudios más integrales y flexibles con énfasis en los aspectos metodológicos de la producción del conocimiento, su manejo y extensión. Se aprecia una transición desde una especialización temprana en las primeras carreras, a una especialización más tardía que habilite una formación generalista e integral primero y luego un período de especialización que puede ser continuada en el postgrado. 
En 1980 se realiza el último cambio curricular al crear cuatro carreras de Biología con las orientaciones en Ecología, Botánica, Zoología y Paleontología. Posiblemente, esta división fue impulsada por la tradición previa a 1978 donde las carreras eran de Ecología, Botánica, Zoología y Paleontología. Este nuevo plan podría interpretarse como una combinación entre el esquema de planes separados disciplinarmente y un plan de Biología integral. En la figura 2 se muestra el esquema curricular de los planes de estudios de las carreras de Biología de 1980.

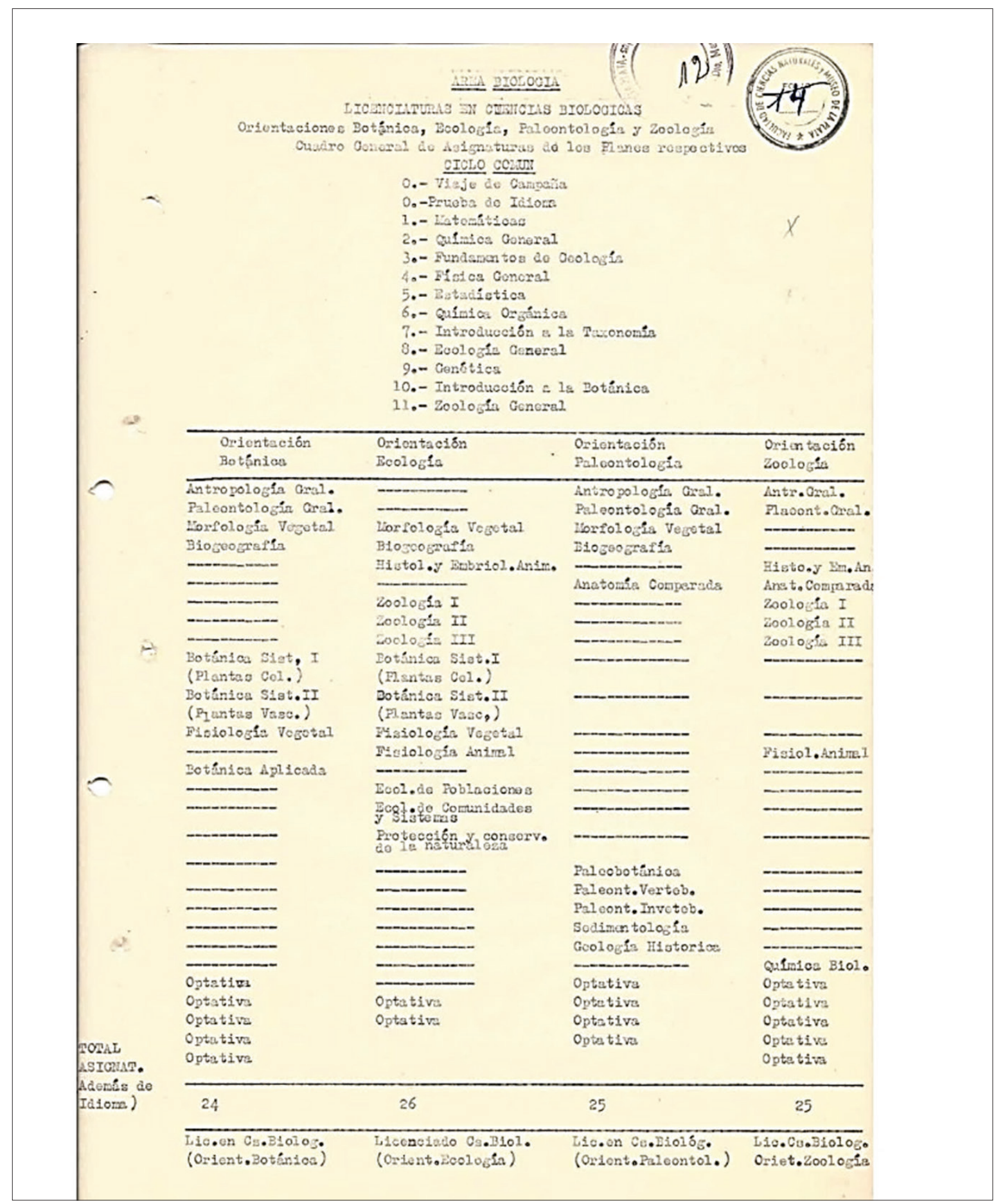

Figura 2: Esquema de los planes 1980 para las licenciaturas en Biología de la FCNyM. Fuente: Archivo de la FCNyM. 
El expediente que trata estos planes de estudios se conforma por una breve introducción a los cambios realizados, el perfil de las carreras, el listado de las materias, las correlatividades y algunos aspectos reglamentarios básicos. En estos documentos institucionales no se establecen cargas horarias ni para las materias ni para las carreras y solo se manifiesta que la duración de las carreras debe ser de al menos 3500 horas. Esta ausencia no es un error sino una práctica aceptada en esos años, pero que con el transcurso de las décadas ha colaborado para que las materias puedan sumar contenidos y por ende carga horaria de manera arbitraria, sólo a veces limitadas por lo estipulado en los reglamentos de aplicación de los planes que corren por expedientes paralelos al plan de estudios.

Si bien en el imaginario institucional, docente y del alumnado, las carreras de Biología de la FCNyM tienen un perfil predominantemente científico, en el currículo no se aprecian espacios específicos para desarrollar las habilidades que requiere la investigación científica. Sólo se encuentran actividades circunscritas a trabajos prácticos en cátedras como Ecología de Poblaciones, Estadística, Paleozoología y Evolución (entre otras), que se aproximan a un desarrollo de las metodologías de la investigación científica. Adicionalmente, el abordaje de los aspectos epistemológicos de la ciencia resulta escaso en algunas orientaciones.

Esta fuerte contradicción entre lo que se supone que las carreras de Biología ofrecen en la FCNyM y el tipo real de formación que los estudiantes recorren, pone a la institución en la obligación de revisar y replantear los planes de estudios y las prácticas de enseñanza en el corto plazo. El desarrollo de espacios formalizados para el aprendizaje epistemológico y metodológico, relativo a las prácticas profesionales, incluso en la investigación científica, debe ser uno de los principales ejes de innovación académica.

\section{El desarrollo de espacios formalizados para el aprendizaje epistemológico y metodológico, relativo a las prácticas pro- fesionales, incluso en la investigación científica, debe ser uno de los principales ejes de innovación académica.}

\section{LOS ACTORES Y DISCURSOS DEL CAMBIO CURRICULAR}

Es en los últimos 10 años que los sujetos y condiciones institucionales y externas están siendo adecuados para que el cambio curricular ocurra en la FCNyM. Como expresan Havelok y Huberman (1980: 45), los sistemas cambian cuando ocurre un hecho que altera el equilibrio, esto puede tratarse de crecimiento o de decadencia. En el juego de poderes prevaleció durante tres décadas la cuasi-inmutabilidad formal, hasta que la acumulación de tensiones con la propia realidad de 
cada uno de los sujetos (entendidos aquí como colectivos sociales) desencadenó en el enorme desafío institucional de transformar los currículos, en un contexto de fuertes contrapuntos y consensos en una institución diversa y compleja como lo son todas las instituciones universitarias. Esto es el reflejo de que un cambio curricular es en esencia un cambio cultural de la comunidad que lo desarrolla (Coscarelli, 2017: 32). Es en este sentido que la realización de una sistematización de la experiencia de las instituciones universitarias en sus procesos de cambio curricular, permite volver evidente las confluencias y las divergencias, los consensos y los disensos, de manera que las experiencias dejen de ser solo información para convertirse en conocimiento.

Resulta esencial en el proceso de sistematización del cambio de planes de estudios poder identificar y analizar los diferentes actores curriculares que forman parte del proceso de negociación y elaboración de esos documentos (De Alba, 1995: 67). En la FCNyM han participado tres formas de espacios: a) formales y representativos (Claustro de profesores, Consejos Consultivos Departamentales, Comisiones Asesoras y Consejo Directivo), b) asambleas (asambleas de estudiantes y graduados, Jornadas Institucionales) y c) núcleos informales (agrupaciones informales de miembros de la comunidad académica con afinidades en los objetivos y metodologías).

\section{Resulta esencial en el proceso de sistematización del cam- bio de planes de estudios poder identificar y analizar los diferentes actores curriculares que forman parte del proceso de negociación y elaboración de esos documentos}

A lo largo de los años la participación ha sufrido altibajos, lo cual ha tenido como consecuencia directa la inmovilización del proceso de cambio curricular. Muchos de quienes en algún momento tuvieron participación activa, frustrados ante la lentitud del proceso y los continuos retrocesos, han abandonado su intervención. La dinámica de los espacios representativos hace que los actores o referentes de cada claustro cambien periódicamente y esto aporta a la pérdida de continuidad del proceso. La incapacidad de cumplir con cronogramas de trabajo por la falta de participación y continuidad llevó a la discusión y rediscusión de los mismos temas en donde lo único que variaba eran los interlocutores. Este desgaste acentuó las diferencias entre los claustros y la dilación de definiciones que permitieran avanzar en el proceso de cambio curricular.

Los ámbitos disciplinares donde se han discutido los cambios curriculares son los Consejos Consultivos Departamentales (CCDs), uno por cada carrera de Biología, espacios cogobernados integrados por los tres claustros académicos (estudiantes, graduados y profesores). El principal aporte de los CCDs fue la discusión y elaboración de los contenidos mínimos y objetivos de cada espacio curricular, teniendo en cuenta los perfiles de las carreras. Como muchas materias son comu- 
nes entre las carreras, sus enfoques y contenidos fueron elaborados con la intervención de los CCDs respectivos.

El órgano representativo coordinador de los procesos de cambio curricular en el FCNyM es la Comisión Asesora de Planes de Estudios (CAPE) del Consejo Directivo. En la composición de la CAPE se incorporan miembros de cada área para disponer de la experiencia y representatividad requerida para el tratamiento de los planes de estudios. Aunque esto ha sido valioso desde el aporte que cada disciplina puede realizar al resto, también actuó como condicionante en el tratamiento y toma de decisiones que involucran aspectos más específicos de los planes. En este sentido, resultaba difícil tomar decisiones sobre un plan de estudios, por ejemplo de la orientación Paleontología, cuando solo se contaba con un solo miembro de ese área en la CAPE. Es por esta razón que la comisión debió ir ajustando el tipo de intervención que podía realizar y adquirió recién en los últimos años el perfil de coordinadora y revisora del trabajo realizado por los CCDs, cuya especificidad disciplinar permite abordar esta problemática. En este contexto, la comisión se abocó a la producción de documentos resúmenes, documentos orientativos, asesoramiento e información a los CCDs, organización de jornadas institucionales y recientemente la creación de una sección sobre el cambio de planes de estudio en la página oficial de la Facultad.

Lejos de agotar el análisis de estos contextos, interesa focalizar un aspecto que tuvo una incidencia importante en los debates curriculares de la formación en Biología en el país. En el año 2011 las carreras de Biología y afines fueron declaradas de interés nacional bajo la resolución ministerial No 139/2011. De esta manera, las carreras de Biología entran en la regulación del artículo 43 de la Ley de Educación Superior (Ley $N^{\circ} 24.521$ ), lo que implica que las mismas deben ser evaluadas y acreditadas por la CONEAU. Los estándares de evaluación responden a criterios de calidad académica mínimos definidos por el Ministerio de Educación de la Nación a propuesta del CIN (Consejo Interuniversitario Nacional) que a su vez utiliza como base lo acordado por el Consejo Interuniversitario para la Enseñanza Superior de la Biología (CIPEB).

La evaluación externa en una primera instancia representó una dificultad para el proceso de cambio curricular, dado que el claustro de estudiantes asociaba a la CONEAU con políticas neoliberales impuestas durante la década de los 90 . Sin embargo, a medida que se desarrolló el proceso de evaluación de las carreras vigentes y se percibió que los informes realizados por los pares evaluadores coincidían con las apreciaciones que la mayor parte de la comunidad académica local tenía de las carreras, la intervención de la CONEAU sirvió como catalizador de los procesos de debate internos de la Facultad. Esta intervención externa tuvo como consecuencia la aceleración y el incremento de la participación de referentes de los distintos claustros y actores institucionales. Los requerimientos realizados por los pares evaluadores de la CONEAU aportaron definición en las tensiones que ya existían en el proceso de cambio curricular de las carreras de biología de la FCNyM. 
El claustro que mayor nivel de participación tiene en las convocatorias de discusión es el de estudiantes. Los estudiantes son los que están "transitando" los planes de estudios vigentes y se han manifestado críticos no sólo por la falta y/o exceso de algunas temáticas en los planes de estudios, sino también en la estructura e implementación de éstos. En este sentido, los principales reclamos de los estudiantes son el exceso de carga horaria semanal que asciende hasta las 45 hs. de cursada (considerando clases teóricas y prácticas), el gran número de correlatividades entre las asignaturas que a su vez varían dentro de las carreras dependiendo de la orientación y la ausencia de bandas horarias.

A pesar de que los estudiantes tienen un buen nivel de participación, éste no se consolida en un aporte continuo dada la movilidad que tienen sus representantes en los espacios formales de discusión y elaboración. Las posturas de algunos referentes estudiantiles anclan a la CONEAU con reformas y debates que se dieron en la década del 90 sobre la Educación Superior. En este sentido, desde algunos sectores estudiantiles ha habido intentos de deslegitimar el proceso de cambio en tanto interpretan que es más el producto de demandas externas que de necesidades internas.

Desde hace aproximadamente seis años, los representantes del claustro de estudiantes han ido integrándose progresivamente a la discusión y elaboración de los planes de estudios desde una postura más constructiva. Las posibles razones de este cambio en la concepción del proceso de cambio curricular podrían buscarse en dos circunstancias: un cambio generacional de la conducción estudiantil, antes relacionada directamente con la lucha contra la Ley de Educación Superior (LES), y la organización de comisiones ad hoc de estudiantes para el análisis de los documentos institucionales, la discusión y la elaboración de propuestas propias presentadas en los ámbitos formales.

La participación de los profesores de la Facultad en el proceso de reforma curricular de Biología puede describirse como discontinua. Tan solo una docena de docentes ha tenido continuidad y ha impulsado a lo largo de más de 15 años los avances que se pueden registrar en el diseño de los nuevos planes de estudios. Cada vez en menor proporción, pero aún presentes, existen docentes que apelan a la tradición naturalista de la Facultad y la fuerte articulación que tiene con el Museo de La Plata como argumento para no modificar los planes de estudios. Por otra parte, y como en todo proceso de cambio curricular, se manifiestan preocupaciones concretas en el plantel docente respecto a su futura condición laboral. Esta incertidumbre varía de un docente a otro, siendo en unos admitida como parte de la evolución de la institución, y en otros como un ataque directo a su posición de poder dentro de la misma. La incertidumbre de las condiciones laborales y/o la inseguridad que deriva de modificar el status quo, son cuestiones de difícil lectura y por ende de compleja resolución porque comprenden aspectos personales de los involucrados. Existen trayectorias laborales a las que es necesario atender en la propuesta institucional de reflexión y toma de 
decisiones. Esto configura otra de las dimensiones que aporta complejidad a los procesos de cambio curricular en el espacio universitario.

El claustro de graduados podría ser descripto como el más heterogéneo. Está integrado por graduados que son docentes de la Facultad, con un mayor grado de conocimiento sobre la misma por estar laboralmente insertos en ella, y por graduados que haciendo investigación en otra institución o ejerciendo otra práctica profesional están menos vinculados a las problemáticas actuales de la Facultad. El hecho que los graduados tengan espacios de desempeño diversos, en comparación con el claustro de profesores y estudiantes, tiene como efecto una menor comunicación e interacción hacia el interior del claustro y también para con el resto de la comunidad académica.

Desde el Centro de Graduados de la Facultad se han realizado numerosas convocatorias para integrar este claustro a la discusión de los planes de estudios. Sin embargo, las reuniones sobre planes de estudios no superan la docena de graduados, y en general en los sucesivos encuentros disminuyen. Durante los últimos años, gracias a la activa participación de las agrupaciones de graduados de la Facultad, el tema de los planes de estudios ha vuelto a ser tema de discusión entre los graduados. Aunque con posturas a veces contrapuestas, las agrupaciones de graduados han estimulado la participación del resto del claustro a través de reuniones, informes y encuestas.

Es posible sostener que el proceso de cambio curricular de las carreras de Biología conforma la innovación pedagógica más prolongada y compleja de la que la FCNyM ha sido protagonista. La variedad de experiencias documentadas y no documentadas que surgieron durante este trayecto requieren ser sistematizadas con el fin de volver evidentes elementos implícitos, reflexionar sobre las principales decisiones tomadas en el camino y dar la posibilidad de que futuras modificaciones cuenten con un relato pormenorizado y crítico de esta experiencia. Los cambios socio-históricos que atraviesa la trayectoria de una institución pueden ser enriquecidos si se van creando cuerpos documentales de experiencias que robustecen el quehacer de los nuevos protagonistas. La sistematización en el campo pedagógico permite no solo la organización cronológica de eventos e información, sino también analizar desagregadamente las fases y protagonistas del proceso con un enfoque histórico que habilita el aprendizaje crítico con el objetivo de mejorar las condiciones de futuros procesos. La sistematización permite interrogar a la experiencia para entender por qué el proceso histórico ocurrió de esa manera y no de otra, dando lugar a pasar de lo meramente descriptivo a una interpretación crítica (Jara, 2005: 2). las carreras de Biología conforma la innovación pedagógica más prolongada y compleja de la que la FCNyM ha sido protagonista. 


\section{CONCLUSIÓN}

Actualmente el proceso de cambio curricular en las cuatro licenciaturas en Biología de la FCNyM está llegando a su fase final. Las diferentes posiciones descriptas en esta contribución han quedado representadas en propuestas colectivamente construidas que, recuperando la historia de la institución, han permitido al mismo tiempo incorporar los nuevos problemas, saberes y campos de intervención de la Biología. Las nuevas propuestas cambian la lógica histórica de la existencia de materias anuales por la implementación de cursadas cuatrimestrales. Asimismo, la existencia de un ciclo común de un cuatrimestre para las siete carreras de la unidad académica, sostiene la existencia de una identidad propia del programa académico de la FCNyM. A través de este trabajo se ha intentado realizar un aporte al proceso de historización de los sentidos y debates del proceso de este cambio curricular (Autor, 2018: tesis). Aunque este complejo proceso institucional se ha extendido en el tiempo, la posibilidad de que en el 2020 se puedan contar con los proyectos curriculares de las carreras de Biología producto del consenso y de la participación colectiva, consolida la concreción de un nuevo programa académico en la FCNyM. 


\section{BIBLIOGRAFIA}

Burton, R.C. (1991).“El sistema de educación superior: Una visión comparada de la organización académica". Méjico.Editorial: Nueva Imagen.

Autor (2018). “El proceso de cambio curricular en los planes de estudios de las carreras de Licenciatura en Biología en la Facultad de Ciencias Naturales y Museo (UNLP). Perspectiva histórica y crítica". (Trabajo Final Integrador de la Especialización en Docencia Universitaria - UNLP). Recuperado de: http://sedici.unlp.edu.ar/handle/10915/73576

Coscarelli, M. R. (2017). “Currículum e institución. En: Didáctica y currículum: aportes teóricos y prácticos para pensar e intervenir en las prácticas de la enseñanza". Picco, S. y Orienti, N. coordinadoras. La Plata: EDULP. (Libros de cátedra. Sociales). En Memoria Académica. UNLP. Argentina. Recuperado de: http://www.memoria.fahce.unlp.edu.ar/libros/pm.560/pm.560.pdf

De Alba, A. (1995). “Currículo: Crisis, Mito y Perspectivas". Buenos Aires. Miño y Dávila Editores. Argentina.

García, S.V. (2004). "Miguel Fernández y el proyecto científico-educativo del laboratorio de zoología del Museo de La Plata (1906-1926)". Saber y Tiempo. 17: 97-127. Argentina. Recuperado de: http://envios.unsam.edu.ar/publicaciones/Archivos/Saberytiempo17.pdf

Havelock, R.G. Huberman,A.M. (1980). “Innovación y problemas de la educación: teoría y realidad en los países en desarrollo". Unesco. Suiza. Recuperado de: https://unesdoc.unesco.org/ark:/48223/pf0000136018

Jara, O. (2005). “El desafío político de aprender de nuestras prácticas Diálogos: Educación y formación de personas adultas". 2 (42-43): 59-64. Perú. Recuperado de: http://www.cepalforja.org/sistem/documentos/aprenderdepracticas.pdf

Teruggi, M.E. (1994). "Museo de La Plata 1888-1088: Una centuria de honra”. Fundación Museo de La Plata. Tercera edición. 